\title{
Trânsito de suídeos em Minas Gerais, Brasil
}

[Swine industry animal network in Minas Gerais, Brazil]

\author{
C.S.F. Oliveira ${ }^{1}$, J.P.M. Gonçalves ${ }^{4}$, J.B. Utsch ${ }^{3}$, R.R. Nicolino ${ }^{1}$, \\ M.X. Silva ${ }^{3}$, J.P.A. Haddad ${ }^{3 *}$
}

\footnotetext{
${ }^{1}$ Aluno de pós-graduação - Escola de Veterinária - Universidade Federal de Minas Gerais - Belo Horizonte, MG

${ }^{2}$ Aluno de graduação - Escola de Veterinária - Universidade Federal de Minas Gerais - Belo Horizonte, MG

${ }^{3}$ Escola de Veterinária - Universidade Federal de Minas Gerais - Belo Horizonte, MG

${ }^{4}$ Fiscal agropecuário do estado de Minas Gerais
}

\begin{abstract}
RESUMO
Minas Gerais ocupa a quarta posição entre os estados brasileiros com maior produção de suídeos. A suinocultura envolve intenso trânsito de animal e, consequentemente, forma complexas redes de fluxo. Com a movimentação, esses animais podem carrear agentes patogênicos que podem ser disseminados por meio dessa rede de contatos. Diante disso, este estudo tem como objetivo descrever e analisar o trânsito de suídeos em Minas Gerais para compreender os caminhos mais prováveis para a disseminação de possíveis surtos. Os dados foram fornecidos pelo Instituto Mineiro de Agropecuária, originados das 56.823 Guias de Trânsito Animal emitidas no ano de 2009. A movimentação dos 5.354 .735 suídeos foi analisada segundo a sua finalidade. O trânsito mais intenso ocorreu com a finalidade de abate $(79,95 \%)$, seguida pelas finalidades de engorda $(10,64 \%)$ e de reprodução $(9,38 \%)$. A movimentação de animais para exposições e leilões representou apenas $0.03 \%$ dos transportes realizados. O trânsito em Minas Gerais é heterogêneo e concentra-se, principalmente, nas regiões do Triângulo Mineiro, Alto Paranaíba e Zona da Mata. As características do fluxo de animais tornam a utilização da estratégia das redes ideal para a elaboração das medidas de vigilância e controle das doenças dos suídeos em Minas Gerais.
\end{abstract}

Palavras-chave: movimento de suínos, redes de fluxo, saúde animal, grafos

\begin{abstract}
Minas Gerais is the fourth largest Brazilian state in swine production. This activity involves the intense animal movement producing a complex network. In this context animals can carry on infectious agents and transmit them among the population. The aim of this study is to describe and analyze the movement of swine in Minas Gerais and to understand the most likely paths to spread possible outbreaks. Data were provided by the Instituto Mineiro de Agropecuária, derived from 56.823 Transit Animal Guides issued in 2009. The movement of 5.354.735 swine was analyzed according to its purpose. The traffic was more intense for slaughter (79.95\%) followed by finishing (10.64\%) and reproduction purposes $(9.38 \%)$. The fairs and auction purposes represented only $0.03 \%$. Movement of animals in Minas Gerais is heterogeneous and is mainly concentrated in same regions such as Alto Paranaíba and Zona da Mata. It also occurs in the Central and South regions. The network approach, based on graph theory, can be used more efficiently for the development of swine disease control programs and crisis management in Minas Gerais.
\end{abstract}

Keywords: swine movements, networks, animal health, graphs

\section{INTRODUÇÃO}

As atividades relacionadas à suinocultura ocupam lugar de destaque na matriz produtiva do agronegócio brasileiro, destacando-se como uma

Recebido em 12 de março de 2013

Aceito em 2 de setembro de 2013

E-mail: camila.sfo@terra.com.br atividade de relevância nos âmbitos econômico e social. Com quase 40 milhões de suínos, produção de três milhões de toneladas de carne e geração de 630 mil empregos diretos e indiretos e no varejo, é uma importante atividade econômica, principalmente no sul e sudeste do país (Neto, 2009). 
Minas Gerais, o quarto principal plantel do país, possui mais de 5 milhões de cabeças e detém um dos maiores centros industriais de abate do país. Em 2008 produziu cerca de 340 mil toneladas de carne suína (IBGE, 2009).

As concentrações mais elevadas da suinocultura no estado encontram-se na Zona da Mata, no Alto Paranaíba e no Triângulo, em razão da formação de polos especializados nas cidades de Ponte Nova, Urucânia, Patos de Minas, Patrocínio, Piranga e Uberlândia, sendo que o último município citado possui o maior efetivo de suínos do Brasil (IBGE, 2009).

As mudanças na estrutura e nas práticas dos modernos sistemas de produção de suínos têm levado a um aumento tanto do movimento de animais entre granjas quanto da distância percorrida até os abatedouros (Zanella e Duran, 2000).

O trânsito animal está intimamente relacionado à disseminação de doenças. É um fator crítico em todas as epidemias de doenças infecciosas, e o monitoramento dessa movimentação permite reduzir os riscos de introdução ou reintrodução de patógenos (Capanema, 2010).

Uma rede com as rotas do trânsito animal fornece informações sobre a transmissão e a propagação de doenças (Bigras-Poulin et al., 2007). Conhecer essas redes de fluxo pode proporcionar uma melhor relação custo-benefício para as medidas de combate dos programas de saúde animal (Ortiz-Pelaez et al., 2006).

\section{MATERIAL E MÉTODOS}

O presente trabalho é um estudo descritivo do trânsito de suídeos e teve como base o ano de 2009, envolvendo os 1.412 estabelecimentos produtores de suínos do estado de Minas Gerais cadastrados nesse ano.

O trânsito de suídeos consiste na movimentação de um ou mais suínos ou javalis, entre dois estabelecimentos credenciados pelo Ministério da Agricultura Pecuária e Abastecimento (Mapa), para o qual deve ser emitida a Guia de Trânsito Animal (GTA).

Os dados sobre o trânsito de suínos foram gentilmente cedidos pelo Instituto Mineiro de
Agropecuária (IMA), obtidos das GTA(s) emitidas pelo órgão (Minas Gerais, 2009). Esses documentos contêm os registros das movimentações de suínos efetuadas na área e no período de estudo. Foram utilizados a data de emissão, a propriedade e o município de procedência e de destino, o número de animais movimentados, o sexo dos animais, a finalidade da movimentação, o tipo de transporte utilizado, distribuído entre rodoviário, a pé, outros (transporte fluvial ou aéreo) ou desconhecido e o responsável pela emissão.

Todos esses dados presentes nas 56.826 GTA(s) emitidas em 2009 foram cedidos sob forma de uma base de dados unificada, produzida automaticamente após a transmissão dos dados pelo emitente no sistema GeoDSA. Um banco de dados foi recebido, transferido e adaptado para uma planilha de cálculo.

Esse conjunto de dados forma um censo dos movimentos de suídeos registrados, de $1^{\mathrm{o}}$ de janeiro a 31 de dezembro de 2009, não havendo vieses ligados à amostragem, porém nem todos os erros de digitação puderam ser conferidos a fundo, pelo grande volume de informações, sendo este uma possível fonte de erro assumida no trabalho.

Primeiramente, realizou-se uma avaliação da consistência das planilhas com o auxílio da aplicação de filtros de modo a visualizar as informações mais relevantes.

Para a representação espacial, foi utilizado o programa Terraview ${ }^{\circledR}$ 4.0.0 (Instituto..., 2010), em que os dados da análise descritiva foram convertidos para mapas temáticos sob a plataforma de divisão entre municípios do Instituto Brasileiro de Geografia e Estatística IBGE

A representação gráfica das redes de fluxo, ou redes sociais, foi realizada com auxílio do programa Pajek 1.24 (Pajek, 2009).

\section{RESULTADOS E DISCUSSÃO}

O ano de 2009 foi o primeiro ano completo de vigência da obrigatoriedade da emissão eletrônica das GTA(s), realizada exclusivamente pelo software GeoDSA (Gerência de Defesa Sanitária Animal) promulgada pela Portaria no. 
934 de 23 de setembro de 2008 (Minas Gerais, 2008). Isso permitiu maior rapidez na consolidação do banco de dados, além de mais uniformidade nos dados gerados. Porém, a análise de consistência da planilha gerada com todos os dados das GTA(s) do referido ano mostrou que não era possível trabalhar com o trânsito entre propriedades e/ou estabelecimentos, devido a grandes inconsistências nos dados de georreferenciamento quando comparadas aos dados do cadastro de suinocultores do estado de Minas Gerais ou, até mesmo, pela inexistência desses dados geográficos.

Também não foi possível avaliar o fluxo da entrada de suínos em Minas Gerais, devido à ausência de uma base de dados unificada no país contendo as GTA(s) emitidas em todas as unidades federativas (UF) e também pela falta de registros formais da captação dos animais movimentados pelas localidades de destino.

O Serviço de Defesa Animal foi alertado sobre as não conformidades observadas, tendo sido esse trabalho importante para que melhorias fossem realizadas na plataforma do sistema adotado e no treinamento dos profissionais habilitados à emissão das GTA(s), pois a qualidade e a veracidade das informações disponíveis nesse banco de dados são fatores de extrema relevância para uma análise do trânsito precisa e consistente, como observado por Brigas-Poulin et al. (2007) e Ortiz-Pelaez et al. (2006).

Houve em 2009, no estado de Minas Gerais, o trânsito de 5.354.735 suídeos, sendo 708 javalis e 5.354.027 suínos. Para isso, foram emitidas 56.823 GTA(s) com as finalidades de abate, engorda, exposição, leilão e reprodução (Tab. 1).

Tabela 1. Número de GTA(s) por espécie animal e finalidade

\begin{tabular}{lrrrr}
\hline Finalidade & GTA(s) de suínos & GTA(s) de javalis & Total & $\%$ \\
\hline Abate & 45430 & 9 & 45435 & 79.96 \\
Engorda & 6045 & 1 & 6046 & 10.64 \\
Exposição & 2 & 0 & 2 & 0.00 \\
Leilão & 5 & 0 & 5 & 0.01 \\
Reprodução & 5331 & 0 & 5331 & 9.38 \\
\hline Total & 56813 & 10 & 56823 & 100 \\
\hline
\end{tabular}

A análise do trânsito mensal durante o ano de 2009 revelou que o maior fluxo de suínos ocorre no segundo semestre. Em dezembro houve um aumento de $32,1 \%$ no número de animais transportados, com a média de 52.791 animais transportados por dia, enquanto nos demais meses a média mensal variou entre 10.241 (janeiro) e 18.441 (setembro) suídeos. Esse comportamento provavelmente aconteceu devido ao aumento esperado do consumo de carnes e derivados suínos durante as festividades de fim de ano, o que leva a um aumento do abate e da reposição desses animais para engorda.

Ao todo, 99,98\% desses animais foram levados aos abatedouros por meio de transporte rodoviário. Todos os 667 suínos $(0,02 \%)$ tangidos pelo solo no período foram transportados com a finalidade de abate.
Minas Gerais, em 2009, possuía 1.412 granjas suinícolas cadastradas, situadas, principalmente, nas regiões do Triângulo Mineiro, Alto Paranaíba, Sul, Zona da Mata e Central. Características climáticas favoráveis à atividade e a proximidade dos polos de produção de milho e soja levaram à maior concentração e expansão dos estabelecimentos nessas localidades, principalmente na região do Triângulo Mineiro (Garcia, 2011), onde se destaca o município de Uberlândia, detentor do maior rebanho suíno, com 684.810 cabeças, o que representa $1,8 \%$ do rebanho brasileiro (IBGE, 2009).

Os 83 estabelecimentos de abate registrados e inspecionados pelos serviços de defesa sanitária, que abatem suínos, situam-se próximo a essas regiões, em que o rebanho suíno é maior (Fig. 1).

Para a compreensão mais apurada da dinâmica de animais e para o planejamento de estratégias 
de controle e vigilância, é importante que estudos do fluxo de suídeos entre os estabelecimentos ligados à suinocultura sejam realizados, como já observado por Batista e Nunes (2007), Capanema (2010) e Felipe (2010). Animais procedentes de 203 municípios mineiros foram destinados para outros 722 municípios. Entre esses, houve o trânsito interno - trânsito em que o município de origem também é o município de destino - em 58 municípios, o que movimentou 536.331 suídeos.

A movimentação do maior número de suídeos por um único trajeto, em 2009, ocorreu dentro do município de Uberlândia em que 112.500 animais foram enviados para estabelecimentos de abate dentro do próprio município. Esse município também se destacou com o maior número de contatos com outros pelo trânsito de suídeos. Ao todo foram enviados 277.325 suídeos do município de Uberlândia para 280 diferentes municípios e recebidos 1.110.052 suídeos de 187 municípios.

Em 2009, de todos os animais procedentes de municípios de Minas Gerais, estado que faz parte da Zona Livre de Peste Suína Clássica - ZLPSC (Brasil, 2001), 1,88\% foram destinados para 432 municípios de outros 19 estados brasileiros em seis regiões brasileiras (Tab. 2). Para essa movimentação interestadual, foram emitidas 2.902 GTA(s), com média de 34,7 animais por guia. O Rio Grande do Sul foi o estado que mais recebeu suínos mineiros, seguido pelo Paraná e por São Paulo. Esses estados também pertencem à ZLPSC.

Tabela 2. Número de municípios, de GTA(s), de animais por estados que receberam suínos de Minas Gerais em 2009

\begin{tabular}{|c|c|c|c|}
\hline Estado de destino & No. de municípios & No. de GTA(s) & No. de animais \\
\hline Alagoas & 5 & 18 & 232 \\
\hline Bahia & 16 & 73 & 2.790 \\
\hline Ceará & 10 & 15 & 296 \\
\hline Distrito Federal & 4 & 92 & 2.188 \\
\hline Espírito Santo & 41 & 478 & 14.239 \\
\hline Goiás & 37 & 382 & 8042 \\
\hline Maranhão & 3 & 6 & 61 \\
\hline Mato Grosso do Sul & 14 & 53 & 243 \\
\hline Mato Grosso & 11 & 139 & 8.409 \\
\hline Pernambuco & 3 & 3 & 5 \\
\hline Piauí & 2 & 6 & 11 \\
\hline Paraná & 52 & 286 & 6.058 \\
\hline Rio de Janeiro & 28 & 807 & 17.080 \\
\hline Rio Grande do Norte & 1 & 1 & 2 \\
\hline Rio Grande do Sul & 92 & 136 & 18.007 \\
\hline Santa Catarina & 34 & 177 & 8.238 \\
\hline Sergipe & 1 & 1 & 2 \\
\hline São Paulo & 77 & 228 & 14.793 \\
\hline Tocantins & 1 & 1 & 1 \\
\hline Total & 432 & 2902 & 100.697 \\
\hline
\end{tabular}

O trânsito com a finalidade de abate colocou em contato 192 municípios de procedência em Minas Gerais com 216 municípios de destino dentro e fora desse estado. Destacam-se Uberlândia, Pará de Minas, Araguari, Ituiutaba e Patos de Minas como as principais origens, e Uberlândia, Patrocínio, Betim e Pará de Minas como os principais destinos, sendo que Uberlândia e Pará de Minas, os dois principais municípios de destino, possuem cinco importantes frigoríficos, e os outros dois possuem quatro estabelecimentos de abate (Fig. 1).

Para a engorda, o trânsito de suídeos gerou o contato entre 150 municípios de procedência e 163 municípios de destino. Destacam-se Uberlândia, Ituiutaba, Pará de Minas, Araguari e Patos de Minas como as principais procedências, sendo responsáveis pelo envio de $24,5 \%$ de 
suídeos para engorda. Uberlândia, Ituiutaba, Monte Alegre de Minas e Uberaba destacam-se como os principais destinos, tendo recebido $56,22 \%$ dos suídeos transportados para engorda.

Uberlândia também se destaca como o município com o principal centro de engorda do estado, tendo sido responsável pela captação de $27,49 \%$ desses suídeos, e foi responsável pelo envio de $9,48 \%$ desses animais para engorda em 2009. O município de Pará de Minas, com a captação de $7,66 \%$, ocupa a segunda colocação no ranking dos principais municípios de destino com a finalidade de engorda de Minas Gerais, seguido por Ituiutaba e Araguari, que são responsáveis pela captação de, respectivamente, $5,9 \%$ e 5,46\% dos suídeos transportados com essa mesma finalidade.

Patos de Minas e Uberlândia se destacaram como os principais municípios de destino para reprodução, responsáveis pela captação de, respectivamente, $22,62 \%$ e $10,58 \%$ dos animais. Em seguida, estão os municípios de Varjão de Minas (5,82\%), Monte Alegre de Minas (5,17\%), Curitibanos $(4,39 \%)$ e Rio Verde $(3,77 \%)$.

Somaram-se $61.403 \quad(53,67 \%) \quad$ suídeos produzidos em Minas Gerais, que foram captados por 19 outros estados brasileiros com a finalidade de reprodução, para os quais foram emitidas 1.609 GTA(s) destacando-se Santa Catarina, Goiás e Mato Grosso, que receberam, respectivamente, $13,41 \%, 12,68 \%$ e $7,34 \%$ desses animais. Foi observado que Santa Catarina, Paraná e Rio Grande do Sul, estados que possuem os maiores rebanhos suídeos do Brasil, receberam $24,62 \%$ dos animais para reprodução enviados por Minas Gerais.

Em 2009, o trânsito de suínos foi feito por 9.548 diferentes trajetos. A atividade de suinocultura distribuiu-se de forma heterogênea em Minas Gerais, porém todos os municípios em que há atividade de suinocultura no estado mostraram ligação por meio do trânsito de suídeos para alguma das finalidades observadas, não tendo sido encontradas redes totalmente isoladas, que estariam mais protegidas ou seriam limitantes para possíveis surtos, como descrito por Friedman e Aral (2001).

As ligações podem ser caracterizadas como mais ou menos intensas de acordo com o número de animais transportados de um local ao outro. Essa intensidade das interações pode favorecer ou desfavorecer a transmissão de infecções (Keeling, 2005).

A aplicação das redes de fluxo ao trânsito de suídeos permitiu reconhecer as rotas mais importantes e diferenciá-las daquelas esporádicas, pois a heterogeneidade na movimentação é considerada. Estudos desse tipo permitem, por exemplo, a utilização de ferramentas como a restrição de alguns contatos dentro da rede para a redução da propagação de uma epidemia (Pena, 2011)

Existem modelos matemáticos sobre a propagação de doenças, como os descritos por Moore e Newman (2000) e por Schoenbaum e Disney (2003). Tais modelos se baseiam num algoritmo que considera o tamanho da propriedade uma constante de movimentação de animais e, com isso, define uma área máxima aonde os animais podem chegar. Esses autores verificaram que são modelos eficientes para simulações, o que permite uma preparação das ações antes da crise, porém pressupõe uma homogeneidade na movimentação animal, o que não ocorre com o trânsito de suídeos em Minas Gerais. Isso torna tais modelos pouco funcionais diante de uma real crise.

A rede de fluxo de suínos para abate foi composta por 5.937 diferentes trajetos (Fig. 2) em 2009, com média de 419,48 km por trajeto. A maior distância $(1.724 \mathrm{~km})$ ocorreu entre os municípios Ituiutaba e Simões Filho, na Bahia. Cerca de $75 \%$ do trânsito para abate ocorreu nas regiões do Triângulo Mineiro e da Zona da Mata.

A rede de fluxo de suínos para engorda é composta por 1.835 diferentes trajetos (Fig. 3), com média de $472,86 \mathrm{~km}$ por trajeto. A maior distância $(2.132 \mathrm{~km})$ ocorreu entre os municípios Abre Campo, em Minas Gerais, e Lucas do Rio Verde, no Mato Grosso. 


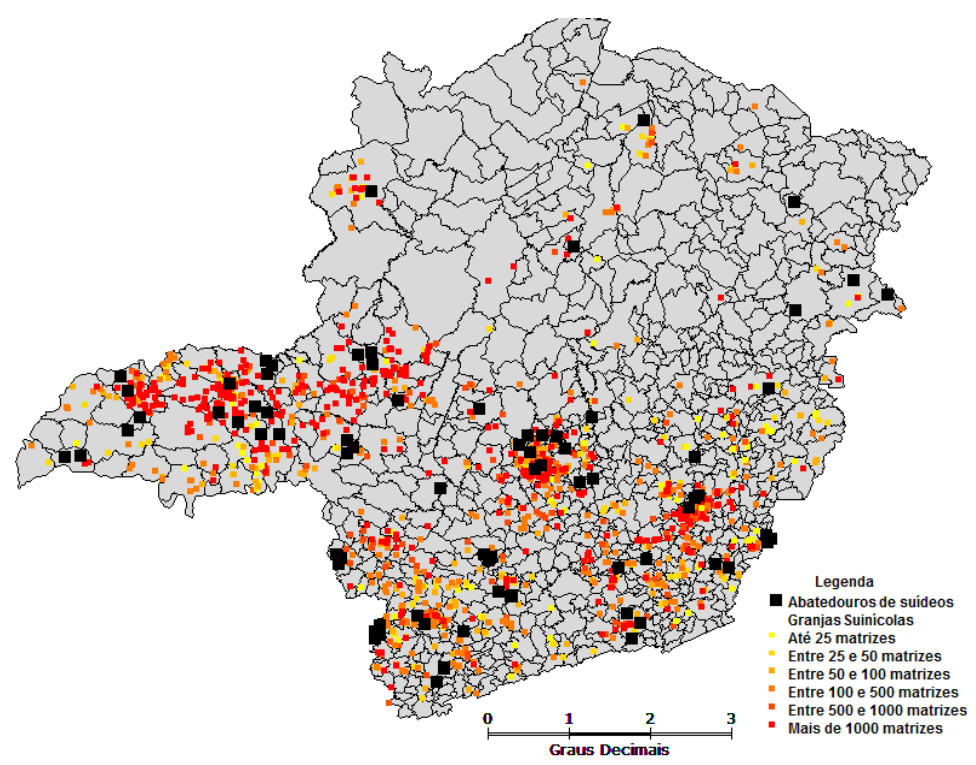

Figura 1. Localização das granjas e dos estabelecimentos de abate de suínos, cadastrados em 2009, em Minas Gerais (Minas Gerais, 2009).

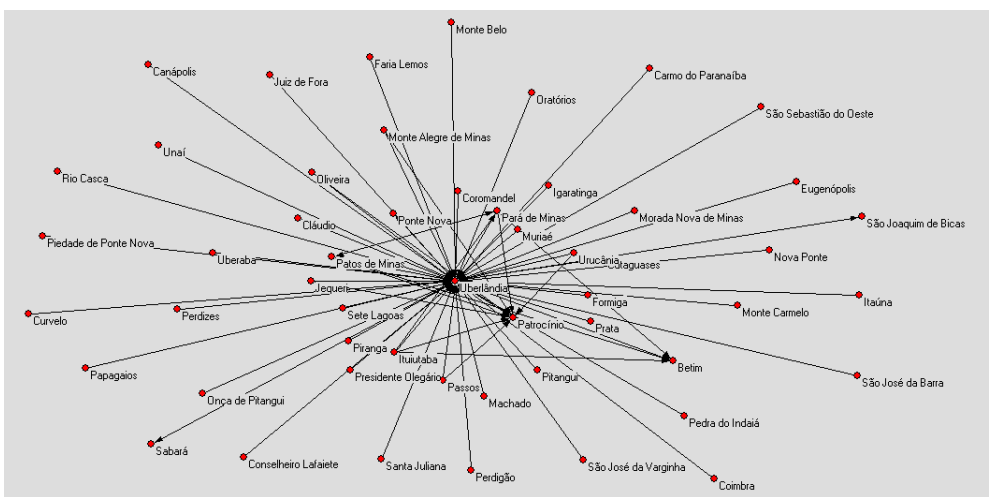

Figura 2. Rede de fluxo de suínos com a finalidade de abate (removidos os $1^{\circ}, 2^{\circ}$ e $3^{\circ}$ quartis $-75 \%$ dos municípios com menor trânsito de suínos) em Minas Gerais em 2009.

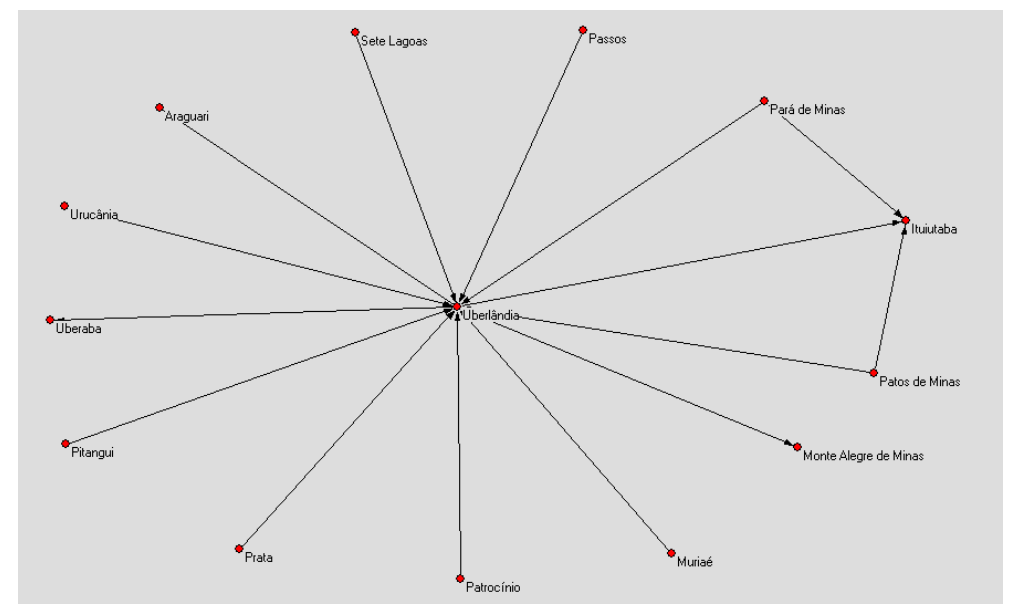

Figura 3. Rede de fluxo de suínos com a finalidade de engorda (removidos os $1^{\circ}, 2^{\circ}, 3^{\circ}$ quartis $-75 \%$ dos municípios com menor trânsito de suínos) em Minas Gerais, em 2009. 
A rede de fluxo total de suínos movimentados com a finalidade de reprodução em 2009 constitui 3.038 diferentes trajetos. Notou-se que poucos municípios (151) enviaram animais para reprodução em um grande número de municípios (551). Foi observada uma média de $535,8 \mathrm{~km}$ por trajeto e distância máxima de $2.192 \mathrm{~km}$ percorridos no trajeto entre Pará de Minas e Teresina no Piauí.
No grafo referente aos $25 \%$ de municípios com maior fluxo de suínos para reprodução (Fig. 4), um circuito bem definido é revelado. Paraúna envia suínos para Unaí e Patrocínio. Este último recebe animais também de Unaí, Ituiutaba, Monte Alegre de Minas e Paraúna. Essa observação revela que esses municípios foram os principais responsáveis pela genética de suínos no estado em 2009.

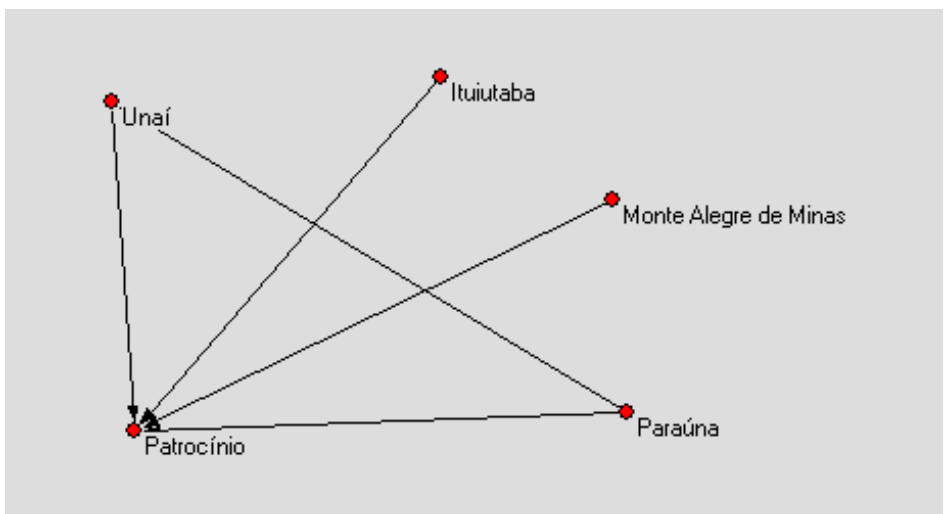

Figura 4 - Rede de fluxo de suínos com a finalidade reprodução (removidos os $1^{\circ}, 2^{\circ}, 3^{\circ}$ quartis $-75 \%$ dos municípios com menor trânsito de suínos) em Minas Gerais, em 2009.

\section{CONCLUSÕES}

O trânsito de suínos em Minas Gerais é intenso nas regiões em que a suinocultura é uma importante atividade (Triângulo Mineiro, Alto Paranaíba, Sul, Zona da Mata e Central). Por isso, gera redes de fluxo bem complexas nessas localidades. Em outras regiões de Minas Gerais, a movimentação é nula. Essas diferenças caracterizam o trânsito de suídeos nesse estado como heterogêneo.

A estratégia de redes é potencialmente uma forma mais eficiente para gestão de crises epidêmicas e para criação de estratégias de vigilância de doenças infecciosas de suídeos no estado de Minas Gerais, pois considera a heterogeneidade do trânsito animal.

Os novos métodos apresentados neste estudo tornam possíveis melhorias nas estratégias de controle e vigilância do estado de Minas Gerais, principalmente se forem integrados a um Sistema de Informação Geográfica (SIG) e se houver dados satisfatórios para análise do fluxo de suídeos entre propriedades e também do fluxo de entrada de suídeos em Minas Gerais.

\section{REFERÊNCIAS}

BAPTISTA, F.M.; NUNES, T.T. Spatial analyses of cattle movement patterns in Portugal. Vet. Italiana, v.43, p.611-619, 2007.

BIGRAS-POULIN, M.; BARFOD, K.; MORTENSEN, S. et al. Relationship of trade patterns of the Danish swine industry animal movements network to potential disease spread. Prev. Vet. Med., v.80, p.143-165, 2007.

BRASIL. Ministério da Agricultura e do Abastecimento. Instrução Normativa n. 01 de 4 de janeiro de 2001. Declara composição da região considerada como Zona Livre de Peste Suína Clássica. Diario Oficial da União de16 de janeiro de 2001, Seção 1, p.6.

CAPANEMA, R.O. Trânsito de bovinos nos Estados do Mato Grosso e Mato Grosso do Sul, Brasil, 2008.2010. 48f. Dissertação (Mestrado em Ciência Animal) - Escola de Veterinária, Universidade Federal de Minas Gerais, Belo Horizonte.

FELIPE, P.L.S. Caracterização do trânsito de bovinos nos Estados do Paraná e Santa Catarina, Brasil, 2008. 2010. 52f. Dissertação (Mestrado em Ciência Animal) - Escola de Veterinária, Universidade Federal de Minas Gerais, Belo Horizonte. 
FRIEDMAN, S.R.; ARAL, R. Social networks, riskpotential networks, health, and disease. J. Urban Health, v.78, p.411-418, 2001.

GARCIA, S.K. Dinâmica e Tendências $d a$ suinocultura mineira em 2009. Material de divulgação do Instituto Mineiro de Agropécuária. Disponível em: imanet.ima.mg.gov.br/nova/gda/.../projetosuinocultura .pdf. Acessado em: 10 jun. 2011.

IBGE - Instituto Brasileiro de Geografia e Estatística. Censo Agropecuário de 2009. Disponível em: http://www.ibge.gov.br/estadosat/temas.php?sigla=mg \&tema $=$ censoagro. Acessado em: 13 nov. 2010.

INSTITUTO Nacional de Pesquisas Espaciais-INPE. TERRAVIEW $^{\circledR}$ Versão 4.0.0. Software gratuito. Disponível em: http://www.dpi.inpe.br/terraview, 2009. Acessado em: 13 de nov. 2010.

KEELING, M. The implication of network structure for epidemics dynamics. Theor. Popul. Bio., v.67, p.18,2005

MINAS GERAIS. Instituto Mineiro de Agropecuária IMA. Portaria no. 934 de 23 de setembro de 2008. Disciplina a emissão de Guia de Trânsito Animal - GTA eletrônica para aves e suídeos no Estado de Minas Gerais. Disponível em: WWW.ima.mg.gov.br/components/search/?searchwor $\mathrm{d}=$ portaria\&ordering $=$ newest\&searchphrase+all\&limit $=100$. Acessado em: 10 jun. 2011 .

MINAS GERAIS. Instituto Mineiro de Agropecuária IMA. Banco de Dados do Cadastro de Suinocultores do Estado de Minas Gerais. 2009. Disponibilizado especialmente para a execução desse trabalho, por meio de arquivo eletrônico, após autorização do Instituto Mineiro de Agropecuária em dezembro de 2010.
MOORE, C.; NEWMAN, M.E.J. Epidemics and percolation in small-world networks. Phys. Rev., v.61, p.78-82, 2000.

NETO, P. C. O desafio de ampliar o mercado externo. Abipecs. Material de divulgação da ABIPECS, 2009. Disponível em: http://www.abipecs.org.br/news/62/97/O-desafio-deampliar-o-mercado-externo.html. Acessado em: 13 de novembro de 2010 .

ORTIZ-PELAEZ, A.; PFEIFFER D.U; SOARESMAGALHÃES R.J. et al. Use of social network analisys to characterize the pattern of animal movements in the inicial phases of the 2001 foot and mouth disease (FMD) epidemia in the UK. Prev.Vet. Med., v.76, p.40-55, 2006.

PAJEK Versão 1.24. Aplicativo para geração e análise de redes de fluxo. Slovenia: Batagelj and Mrvar, 2006.

PENA, C.S. Análise das Redes de Trânsito Animal Integrada à Simulação da difusão de enfermidades infecciosas. 2011. 136f. Dissertação (Mestrado em Estatística) - Instituto de Ciências Exatas, Universidade Federal de Minas Gerais, Belo Horizonte.

SCHOENBAUM, M.A., DISNEY, W.T. Modeling alternative mitigation strategies for a hypothetical outbreak of foot-and-mouth disease in the United States. Prev. Vet. Med., v.58, p.25-52, 2003.

ZANELLA, A.J.; DURAN, O. Bem-estar de suínos durante o embarque e o transporte: Uma visão americana. In: CONFERENCIA INTERNACIONAL VIRTUAL SOBRE QUALIDADE DE CARNE SANTA CATARINA. Anais... Santa Catarina. 2000. 252p. Disponível em: http://www.cnpsa.embrapa.br/ $\mathrm{sgc/sgc}$ _publicacoes/anais00cv_portugues.pdf. Acessado em: 12 de março de 2011 